\title{
Could a single blood test replace TRUS for predicting prostate cancer risk?
}

$\mathrm{P}$

revious studies have shown that a statistical model based on a panel of kallikrein markers in the blood (including total, free, and intact PSA, as well as kallikrein-related peptidase 2) can predict prostate cancer on biopsy. This panel could be a valid alternative for clinical measures such as digital rectal examination (DRE) and transrectal ultrasonography (TRUS), according to a new study performed by collaborators from the USA, the Netherlands, Sweden, Finland, and the UK.

Carlsson et al. evaluated previously unscreened men in the Rotterdam arm $(n=2914)$ and the Göteborg $\operatorname{arm}(n=740)$ of the European Randomized Study of Screening for Prostate Cancer (ERSPC) who underwent sextant biopsy in response to elevated serum PSA levels ( $\geq 3 \mathrm{ng} / \mathrm{ml}$ ). A statistical model based on kallikrein markers was compared with a model based on clinical tests, including DRE and TRUS.

\section{4 ...a noninvasive test- one single blood draw-could possibly replace DRE and TRUS... 77}

The researchers found that prostate volume estimates calculated using the kallikrein panel correlated strongly with TRUS-estimated prostate volumes (Spearman's correlation coefficients of 0.60 and 0.57 in Rotterdam and Göteborg, respectively), and showed that clinical tests (DRE and TRUS) were no more accurate at predicting prostate cancer risk at biopsy than the blood markers. For the outcome of any cancer, a model based on age and kallikrein panel had an area under the curve (AUC) of 0.766 and 0.809 in Rotterdam and Göteborg, respectively (0.847 and 0.786 , respectively, for highgrade disease). AUCs for predicting any cancer and high-grade cancer were very similar when a clinical model (including age, PSA, DRE, and TRUS-estimated prostate volume) was applied. Furthermore, although adding DRE or DRE plus TRUSestimated prostate volume to blood tests improved the discriminatory ability of the panel of blood markers, this effect was minimal.

In terms of the individual kallikreins included in the panel, nicked PSA and free PSA demonstrated the greatest independent correlations with prostate volume in the Rotterdam (Spearman's correlation coefficient of 0.55 ) and Göteborg (Spearman's correlation coefficient of 0.51 ) cohorts, respectively. This finding is consistent with previous studies to show that free PSA and nicked PSA are the most important predictors of prostate transition zone volume.

"DRE and TRUS are invasive tests that require an extra visit to the urologist," says Sigrid Carlsson, a urologist at Memorial Sloan-Kettering Cancer Center who led the study. "What is exciting about our study is the indication that a noninvasive test-one single blood draw-could possibly replace DRE and TRUS as part of the screening procedure, evaluating the indication for biopsy."

When asked what her advice would be to urologists and general practitioners who are asked about this study by their patients, Carlsson stressed that the investigators did not wish to discourage the use of DRE and TRUS, which can be helpful tools in other clinical contexts. "Whether a clinical approach with DRE and TRUS can be replaced by laboratory analyses, or used in combination with other decision models such as risk calculators, is a clinical judgement that will vary from clinician to clinician, depending on how they weigh the different advantages and disadvantages (harms, costs, time, and invasiveness) of both approaches," she explained. limitation of their study relates to the fact that sextant biopsies were used during the first round of the screening trial, whereas biopsies involving 10-12 cores (sometimes as many as 70) are more commonly used today. However, Carlsson and her colleagues also note that the kallikreins measured in their study were taken from frozen and rethawed samples, rather than from fresh blood. Freezing, storage, and thawing of samples are known to affect the stability of free PSA in serum, potentially reducing the predictive accuracy of the model. Thus, it is possible that these effects might counterbalance one another.

It seems reasonable, therefore, to agree with the team's conclusion that a simple blood test could replace invasive clinical tests such as TRUS as part of the screening procedure for evaluating the indication for biopsy. But how is the panel of kallikrein markers likely to compare to relatively new investigative techniques, such as $3 \mathrm{~T}$ multiparametric MRI scans? "How to best screen for prostate cancer is one of the major questions in urological research today," says Carlsson. "Multiparametric MRI holds great promise. In the future, this technique could be combined with both the PSA test and the kallikrein panel. Prospective trials are underway."

Melanie Clyne

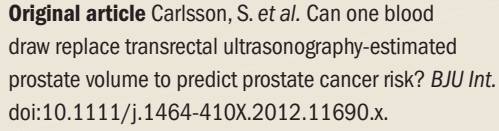

